which the predictor was SE-squared ( $\mathrm{SE}^{2}$; Moreno et al. 2009). In both models, the regression coefficient for the predictor was significant $\left(t_{\mathrm{SE}}=11.87 ; t_{\mathrm{SE}}^{2}=11.99 ; p \mathrm{~s}<.001\right)$, which is consistent with the presence of publication bias. The model-based estimates of the true underlying effect differed, however. Using SEsquared, the corrected effect size was $d=.25$ (95\% CI $[.18, .32])$. Using $\mathrm{SE}$ as the predictor, the corrected effect size was a non-significant $d=-.10(95 \%$ CI $[-.23, .02])$. So, based on these methods, ego depletion could be a small effect-less than half the size of that estimated by Hagger et al.; but it could also be a non-existent effect for which belief has been kept alive through the neglect of null findings. If the true effect size is close to $d=.25$, then the set of experiments Hagger et al. analyzed was extremely underpowered (Mean power $=.15,95$ th percentile $=.24$ ). And even these less skeptical results counsel caution: Assuming the mean effect size is $d=.25$, researchers hoping to study depletion by comparing two means with $80 \%$ power should be prepared to collect a sample with $N>460$, not $N=84$ (as implied by Hagger et al.'s estimate of $d=.62$ ).

The great pity here is that editorial vigilance could have obviated these concerns: Editors and reviewers of meta-analyses should insist on rigorous efforts to track down the hard-to-find (i.e., unpublished) results. As things stand, we believe that the highest priority for research on the depletion effect should not be arriving at a better theoretical account, but rather, determining with greater certainty whether an effect to be explained exists at all.

\section{Can tasks be inherently boring?}

\section{doi:10.1017/S0140525X13000964}

\section{Evan Charney}

Duke Institute for Brain Sciences, Sanford School of Public Policy, Duke University, Durham, NC 27708-0239.

echar@duke.edu

http://www.duke.edu/ echar/

Abstract: Kurzban et al. argue that the experiences of "effort, "boredom," and "fatigue" are indications that the costs of a task outweigh its benefits. Reducing the costs of tasks to "opportunity costs" has the effect of rendering tasks costless and of denying that they can be inherently boring or tedious, something that "vigilance tasks" were intentionally designed to be.

Kurzban et al. begin with the question as to why certain tasks cause an aversive experience of mental effort, with consequent deterioration in task performance. Although almost all of the examples they give are of "vigilance tasks" (to which I shall return below), their answer does not concern such tasks per se, nor does it address the special features of vigilance tasks (or why they are designated "vigilance" tasks in the first place). Rather, Kurzban et al. present a theory about the engagement of executive function in general, along with a general theory of the phenomenology of the conscious experience of "effort," "boredom," and "fatigue." According to the authors, these sensations are an indication of the opportunity costs of an activity namely, that the costs of continued engagement in the present activity are outweighed by the "value of the next-best possible action" (sect. 2.4.1). What is unique about this account is the authors' claim that the costs of an activity simply are its opportunity costs, an assumption that has far-reaching implications.

If the costs of a task are equated with its opportunity costs, then the task itself is costless. Defined this way, tasks cannot be (or be perceived to be) inherently boring or tedious or fatiguing. But this assumption is certainly wrong. Before explaining why, consider the following question: Can tasks be (or be perceived to be) inherently rewarding (or exciting, engaging, invigorating)? The authors do not address this question directly, but it is telling that all of the examples they present of "rewards" or "benefits" associated with a task are external to the task itself - for example, payment given to a study participant for completion of a task. This implies that just as performing tasks is, in and of itself, without cost, so too, performing tasks is, in and of itself, without benefit. Or put another way, just as tasks cannot be inherently boring, they cannot be inherently exciting or rewarding or invigorating. But both of these assumptions (if both are in fact assumptions) are obviously incorrect.

Imagine that you are going to be a study participant. If you were given a choice of two tasks that involved an equal monetary reward and the same amount of time, which would you choose: To watch the second hand on a clock for three hours, indicating its position in response to an irregularly presented signal? Or to pilot a spacecraft simulator through a fantastic 3D virtual reality for three hours? We can safely assume that everyone would choose the latter (and many young persons would likely volunteer to perform the task for free). Why? The answer is not because the opportunity costs are higher for the latter than the former. Presumably, they are the same. Rather, it is because the former task is inherently boring, tedious, and effortful, whereas the latter task is interesting, exciting, and engaging. Can such a claim be made if we accept the authors model? Whether or not they would accept that "benefits" can be internal to, or part of, a task, their model clearly cannot accommodate the claim that watching a clock on a wall is inherently boring. For what could such a claim mean if the costs of a task are reducible to its opportunity costs?

Let us move out of the highly artificial world of study participants being paid to perform tasks like the Macworth Clock for a moment. Let us also consider that "task" is too restrictive a term for what the authors are considering (although appropriate in the context of study participants and Macworth clocks). What they are considering is any kind of mental activity that entails a performance of some sort that can be measured.

Consider two friends, Amy and Peter. Amy is proficient at chess and loves the game. She can play for hours on end with no apparent fatigue and no diminishment in her performance. She is excited and engaged when she plays, and enjoys the experience. Peter is a mediocre chess player and hates the game. He finds it incredibly boring. When Amy insists that Peter play chess with her, Peter has aversive experiences of mental effort, boredom, and fatigue, with a concomitant rapid decline in performance, and he usually forfeits the game after 10 minutes. To be sure, part of Peter's boredom is an acute awareness of opportunity costs - he would rather be doing just about anything else (and is consciously aware of this fact). But the opportunity costs loom large precisely because he finds the activity of playing chess itself boring, tedious, and effortful. In other words, high opportunity costs do not make playing chess effortful and boring for Peter; rather, the fact that Peter finds playing chess boring and effortful makes the opportunity costs high to him. Boredom dictates opportunity costs, not vice versa.

To return to vigilance tasks: While to Peter, in the above example, playing chess may seem a vigilance task, vigilance tasks (unlike chess) are explicitly designed to give rise to aversive experiences of mental effort, boredom, and fatigue, with a concomitant deterioration in performance. That is why they are called vigilance tasks. Interest in vigilance or sustained attention arose during World War II, when British air force radar operators were required to spend lengthy periods of time monitoring screens for the radar return patterns of enemy surfaced submarines (Warm \& Dember 1998). Although the job was considered not physically strenuous or mentally taxing, the maintenance of accurate performance turned out to be beyond human capability. Vigilance tasks were designed to generate the same mental effort, boredom, and fatigue that radar operators indicated that they had experienced.

Finally, concerning the enigma as to why revising a manuscript can be aversively effortful: Many a professor finds writing stimulating, engaging, and exhilarating, and can work for 15 hours without fatigue or boredom. But endless checking of typos, footnotes, and bibliographies bores many to tears, and after an hour or two, just about any activity seems preferable. 


\section{Maximising utility does not promote survival}

doi:10.1017/S0140525X13000976

\section{Daniel B. Cohen ${ }^{a}$ and Lauren L. Saling ${ }^{b}$}

${ }^{a}$ School of Humanities and Social Sciences, Charles Sturt University, Wagga

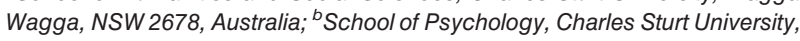
Wagga Wagga, NSW 2678, Australia.

dcohen@csu.edu.au Isaling@csu.edu.au

Abstract: We argue that maximising utility does not promote survival. Hence, there is no reason to expect people to modulate effort according to a task's opportunity costs. There is also no reason why our evaluation of the marginal opportunity costs of tasks should predictably rise with repetition. Thus, the opportunity cost model cannot explain why tasks typically become harder over time.

Kurzban et al. seek to explain two phenomena: (1) why cognitive tasks typically feel increasingly difficult with repetition, and (2) why performance in these tasks degrades with repetition. For instance, in a task involving the continuous multiplication of four-digit numbers over several days, subjects experienced the task as increasingly difficult, and at the same time became less proficient at the task (Arai 1912; Huxtable et al. 1946).

Kurzban et al. offer a common explanation of both target phenomena relying on three claims. They argue: (a) that the difficulty we experience in performing cognitive tasks reflects our subconscious evaluation of the next-best alternative task (this constitutes the marginal opportunity cost of the current task - it is the value we miss out on in virtue of performing that task); (b) that the difficulty we experience in performing cognitive tasks reduces our proficiency in performing those tasks; and (c) that when we repeatedly perform cognitive tasks, our evaluation of the opportunity costs of these tasks steadily increases. These three claims together imply that people who repeatedly perform cognitive tasks will experience increasing difficulty and, as a result, will manifest steadily degraded performance. In our commentary, we wish to challenge claims (a) and (c).

First, what reason is there to think that the difficulty we experience in performing cognitive tasks should reflect our evaluation of their marginal opportunity costs? Kurzban et al. offer an evolutionary answer, arguing that it makes good adaptive sense for an organism to modulate its expenditure of cognitive resources on a task according to the task's expected utility. (Kurzban et al. appear to take the utility of a task to be determined by its contribution to the organism's well-being.) But we question the presupposition that there is any relation between a task's expected utility and its survival value. For example, neither my judgement that rock-climbing is good for me nor the truth of that judgement (entailing that rock-climbing is indeed good for me) implies that this activity is conducive to my survival. It may or may not be the case that our most basic motivation is to maximise utility (our well-being). But even if we are, in fact, utility-maximisers, there seems no reason to think that being a utility-maximiser is adaptively optimal. This point is especially clear if we analyse well-being in terms of preference-satisfaction: There is no reason to assume that the actions that maximise my preferencesatisfaction will also maximise my chances of survival.

But even if we grant the supposition that maximising utility is adaptive, it seems that an organism would benefit most from being disposed, not to modulate its commitment to a task according to the task's expected utility (as Kurzban et al.'s model entails), but rather to fully commit its resources to the cognitive task with the highest expected utility, and to be prepared to shift resources as soon as some other task becomes more beneficial. Thus, even if the value of an alternative task is increasing, it would make no sense for an organism to shift any resources to that task until the tipping point where the alternative task is, in fact, more valuable than the current task. By analogy, if I think Barack Obama is the best candidate, surely I should devote all my resources to his re-election, unless I come to think that Mitt Romney is the best candidate, all things considered. However, I may discover that Romney is a better candidate than I had thought he was-my evaluation of him may indeed be rising; still, this gives me no reason to allocate any resources towards his election until I think he is the best candidate.

Of course, as Kurzban et al. explain in section 2.4.2, it may sometimes be the case that the best task available to an individual involves a sharing of cognitive resources between two activities. In their example, a participant in an Arai-style math task might do best by dividing her "mental processors" between performing the math task and daydreaming. Kurzban et al. argue that as a result of experiencing the math task as effortful, the subject may well end up performing the optimal mixed task of math-plusdaydreaming. However, an optimal mixed task may not always be available. Often, the next-best alternative task will be entirely incompatible with the task at hand. In these cases, according to the model offered by Kurzban et al., people will nevertheless experience aversive effort corresponding to the opportunity costs of their behaviour. For instance, even the subject who performs an optimal math-daydreaming task will, according to the model, continue to experience this task as effortful, corresponding to the value of the next-best task available. But, as we have already argued, there is no benefit in having such a disposition.

Now, what reason is there to think that our evaluation of the marginal opportunity cost of a task will predictably rise when the task is repeated over a brief period of time? In section 2.4.3, Kurzban et al. argue that a bias towards exploring new tasks will protect organisms from over-investing in tasks whose value they overestimate. However, the authors provide no reason for thinking that we are in more danger of overestimating the value of our tasks at hand, rather than alternative tasks. Of course, a task will often seem to lose value with repetition simply in virtue of its increasing sunk costs. But sunk costs should not figure in our calculations about the rationality of a course of action. Ultimately, the account offered by Kurzban et al. in the target article explains one mystery (why cognitive tasks reliably feel harder over time) only by substituting another mystery (why one's evaluation of the marginal opportunity costs of a task should increase - reliably-over time).

\section{An interoceptive neuroanatomical perspective on feelings, energy, and effort}

\author{
doi:10.1017/S0140525X13001489
}

\author{
A. D. Craig \\ Atkinson Research Laboratory, Barrow Neurological Institute, Phoenix, \\ AZ 85013. \\ Bud.Craig@DignityHealth.org
}

Abstract: A homeostatic energy model of awareness proposes that the anterior insular cortex engenders feelings that provide an amodal valuation of homeostatic energy utilization in an opponent, bivalent emotional control system. Feelings are the "common currency" which enable optimal utilization in the physical and mental behavior of a highly social primate. This model offers a different perspective.

As a functional neuroanatomist, I applaud Kurzban et al.'s efforts to integrate parallel literatures, despite the jargon barriers (oh, yes, phenomenology=feelings!). Of course, it's difficult even within our own fields. I'm certain that psychologists who adhere to "self-perception theory" (see Laird 2007) would have difficulty with the statement that "feelings motivate behavior," as do I.

I mapped an ascending neural pathway for affective feelings from the body and identified the cortical image of these elemental feelings in an interoceptive representation of the physiological condition of the body, which anchors the insular cortex of primates (Craig 2002). A convergence of evidence across disparate 